"Seeing the Baby": Pleasures and Dilemmas of Ultrasound Technologies for Prim...

Gillian Harris; Linda Connor; Andrew Bisits; Nick Higginbotham

Medical Anthropology Quarterly; Mar 2004; 18, 1; Health \& Medical Complete pg. 23

\title{
Gillian Harisis
}

School of Social Sciences

University of Newcastle

\section{LINIDA CONNOR}

School of Social Sciences

University of Newcastle

\section{ANDREW BISITS}

School of Medical Practice and Population Health

University of Newcastle

\section{Nick Higginbothiam}

School of Medical Practice and Population Health

University of Newcastle

\section{"Seeing the Baby": Pleasures and Dilemmas of Ultrasound Technologies for Primiparous Australian Women}

\begin{abstract}
The practice of obstetric ultrasound scans has undergone significant expansion in the last two decades and is now a standard part of many women's antenatal care in Australia as elsewhere. This article reviews recent evidence about the value of obstetric ultrasound, summarizing debates and contradictions in research literature and practitioner guidelines. Pregnant women's interpretations of the significance of ultrasound are examined through multiple interviews with 34 study participants. We find that ultrasound has become an integral part of women's embodied experience of pregnancy, with its own pleasures and dilemmas. The increasing use of the technology has augmented the role of scientific biomedicine in the government of pregnancy. This must be understood in the light of trends toward individualized risk management in which the pregnant woman increasingly takes responsibility for the successful outcome of the pregnancy, in a context where pregnancy is discursively constructed as a risky domain of gendered experience in contemporary Australian society. |discourse, obstetric ultrasound, embodiment, pregnancy, feminist |
\end{abstract}

he practice of obstetric ultrasound scans has undergone significant expansion and is now a standard part of many women's antenatal care. Recent research raises questions about ultrasound's efficacy, safety, practice standards,

Medical Anthropology Qutherty 18(1):23-47. Copyright $\bigcirc 20(44$, American Anthropological Association. 
and cost. Feminist analyses of the medicalized management of pregnancy provide insights as to why the expansion of ultrasound scans continues apace, but less has been written about pregnant women's own accounts of ultrasound technology. Davis-Floyd and Sargent argue that technomedicine (as they term it) has its own "fractures and fissures." This is nowhere more evident than in the field of obstetrics, where different types of practitioners contest the value of many practices (Davis-Floyd and Sargent 1997:13). Pregnant women, too, have different expectations and experiences of pregnancy care. Obstetric ultrasound scans are one area of high-technology care that virtually all pregnant women experience in the Australian health care system, as scans have gained a ready acceptance among practitioners and pregnant women alike. However, our qualitative study of pregnant women's concepts of nutrition and well-being suggests that there are dilemmas as well as pleasures associated with ultrasound scans.

We begin this article with a review of evidence about the value of "routine" obstetric ultrasound scans during pregnancy, summarizing the debates and contradictions in research literature and practitioner guidelines. We next discuss the ways in which women themselves interpret the significance of ultrasound in their pregnancies, based on interviews with 34 women. We analyze our results in relation to other studies of routine obstetric ultrasound that have drawn on feminist sociological and poststructuralist perspectives. Emphasized are the ways in which ultrasound has become part of women's embodied experience of pregnancy, its status for women as a source of knowledge about pregnancy, and the nature and extent of criticism about its use by pregnant women.

\section{Development of Obstetric Ultrasound}

Originating as defense and warfare technology, medical "sonar" was first considered as a diagnostic tool in the 1940s. The use of ultrasound as a diagnostic tool developed primarily in the field of gynecology, focusing on the diagnosis of abdominal tumors in women (Levi 1997:485). Focus shifted to pregnant women in the late 1950s (Levi 1997:489), when it was noted that clear echoes could be obtained from the fetal head (Willocks 1996). One of the first obstetrical applications of ultrasound, in 1958, was for the estimation of gestational age (Wade 1999:235). Over the next few years, ultrasound was used for early diagnosis of pregnancy as well as for the diagnosis of multiple pregnancies, placental localization, and fetal abnormality (Willocks 1996).

The development of ultrasound as a method of imaging the fetus coincided with the demise of X-ray due to the hazards brought on the pregnant pelvis. The escalation of the use of ultrasound over the earlier decades is difficult to establish, but can be demonstrated by the virtually complete coverage of pregnant women at the Queen Charlotte Maternity Hospital in London over a ten-year period (1968-78) (Oakley 1986:9). The routinization of the technology is highlighted by a 1978 statement that "ultrasound is now no longer a diagnostic test applied to a few pregnancies regarded on clinical grounds as being at risk. It can now be used to screen all pregnancies and should be regarded as an integral part of antenatal care"(Campbell et al. cited in Oakley 1986:10).

By the 1980s, ultrasound scanning in pregnancy had truly become routine in many affluent industrialized countries. ${ }^{1}$ The number of routine scans differs according 
to national health system context and public versus private funding. The right of pregnant women to two routine ultrasound scans during pregnancy became official policy in Germany in 1980; Austria quickly followed. Recent discussions in the literature indicate a range of scans: one in the United Kingdom, Iceland, and Norway; two in Germany and Canada; and multiples (even every visit) in Greece. ${ }^{2}$ In the United States, despite a statement from the American College of Obstetricians and Gynecologists (ACOG) recommending against routine ultrasound, $\mathrm{ACOG}$ itself has estimated that between 60 and 70 pereent of pregnant women have a "routine" scan (ACOG 1997).

Diagnostic ultrasound is not unique to obstetrics. It is practiced in multiple medical fields, including gynecology, cancer management, and thyroid illnesses. Levi (1997:489) has argued that attributes of obstetrics made it particularly open to the expansion of the technology. In addition to providing a method of imaging the fetus, the expansion was, ironically, due to the fact that the majority of pregnancies are considered "normal" and that all these pregnancies had "something to show." The ultrasound targets (fetus and placenta) generally produced cchoes, as expected.

The richness of obstetrical iconography, due to the large variety and continuous changing of visible structures, meant that it was possible to visibly verify the scanning, given that all examined cases end either in abortion or a birth. The normality of the pregnant body, coupled with the fact the ultrasound targets are conveniently surrounded by amniotic fluid (allowing for clearer interpretation of scans), proved ideal for the development of this new technology. Ultimately, this has enabled the measurement of every part of the fetus and the detailed documentation of changes throughout gestation (Woo 2001). ${ }^{3}$ Technological improvements and increased diagnostic capabilities of scanners have also allowed for the introduction of earlier routine scans. Most recently, attention has been focused on the production of 3-D and 4-D images. ${ }^{4}$

Obstetric ultrasound can readily be interpreted within a feminist narrative of the "technomedical takeover" of pregnancy and childbirth (Davis-Floyd and Sargent 1997:12; Haraway 1991; Jordan 1993; Martin 1987; Oakley 1984; Petcheskey 1987; Wajcman 1994). This narrative examines the way in which late-17th-century medicine, within the context of the Enlightenment and the ascendancy of science, began to develop a more formal and rigid set of institutions. Physicians, who had previously considered the care of pregnant women beneath their dignity, developed the speciality of obstetrics and began to challenge the role of midwives, who were regarded as untrained and uneducated. Male medical hegemony was extended when forceps were introduced in the early 18 th century. According to orthodox medical histories, the use of forceps allowed for the delivery of live babies in cases where mother, baby, or both might previously have died (Happlin 1995). Midwives were precluded from using such instruments, as the access of women to the medical professions was denied (Wajcman 1994:162). The technological innovation of the forceps thus legitimized male physicians' entry to and ultimately control over the previously female domain of midwifery. ${ }^{5}$ Similarly, reproductive technologies that were once welcomed by some feminists as progressive (Firestone 1970) have since been described as aiming to "remove the last woman-centred process from us" (Hanmer 1985; Wajcman 1994). Such technologies, including ultrasound, have been criticized for acting as a powerful means of social control. 


\section{Risks and Benefits: Evaluations of Ultrasound in Pregnancy}

In a review of the history of ultrasound scans in obstetrics and gynecology, medical scientist Joseph Woo summarized the procedure as follows:

Currently used equipments are known as real time scanners, with which a continuous picture of the moving foctus can be depicted on a monitor screcn. Very high lrequency sound waves of between 3.5 to 7.0 megaherts, (i.e., 3.5 to 7 million cycles per second) are generally used for this purpose. They are emitted from a transducer that is placed in contact with the maternal abdomen, and is moved to "look at" (likened to a light shined from a torch) any particular content of the uterus. Repetitive arrays of ultrasound beams scan the fetus in thin slices and are reflected back onto the same transducer. The information obtained from different reflections are recomposed back into a picture on the monitor screen (a sonogram, or ultrasonogram). [Woo 2001:2]

Since the 1990 s, a number of studies of obstetric ultrasound have been carried out internationally, looking at its efficacy as a screening technology, its salety, practico standards and costs. Research lindings about the medical benefits of routine obstetric ultrasound in carly pregnancy (usually less than 20 weeks) are divided. Somo studies show the reduction of perinatal mortality (through termination of pregnancies where fetuses have lethal abnormalities) (Luck 1992; Saari-Kemppainen et al. 1990). Other studies report no effects (Ewigman el al. 1993; Neilson 200(0). Other claimed benefits include the early identification of multiple pregnancy, diagnosis of congenital abnormalities, and more precise estimates of gestational age."

Although the latter findings are supported by a Cochrane Collaboration metaanalysis of eligible studies (Neilson 2000), this review did not substantiate the more significant claims of improved perinatal mortality rates. The Cochrane Review concluded that despite better gestational dating and the earlier detection of twins, neither type of information had been shown to improve fetal outcome. The Cochrane Review (Neilson 20(0):5) also found that the reduction in perinatal mortality demonstrated in the Helsinki Trial (Saari-Kemppainen et al. 1990) was related to an increase in planned pregnancy terminations rather than therapeutic interventions. Overall, the review concluded that larger trials were necessary to demonstrate any real effect.

Safety is another area of contestation. Proponents argue that after 35 years of diagnostic use, ultrasound can be assumed to be safe: "So far, there has been no independently confirmed report that the techniques causes harm to patients" (Kossof 1997:151). In contrast, Enkin et al. conclude:

There has been surprisingly little well-organized research to evaluate possible adverse effects of ultrasound exposure on human fetuses. . . the place of ultrasound for specific indications in pregnancy has been clearly established. The place, if any, for routine ultrasound has not as yet been determined. In view of the lact safety has not been convincingly established, such routine use should for the pre-sent be considered experimental, and should not be implemented outside of the context of randomized controlled trials. [Enkin et al. 1995:44]

Oakley argues that claims to safety are based on the mistaken logic that becausc serious bio-eflects have not been demonstrated, ultrasound is safe (1986:10). It is further assumed that the level of "certainty" about safety increases directly 
with the length of time the technology has been in use, rather than through scrutiny to which the technology has been subjected. She demonstrates the dangers of such assumptions by highlighting the parallels between the use of $\mathrm{X}$-rays and ultrasound in obstetrics. Both procecded before being subjected to controlled trials and both were claimed safe on the basis of the personal conviction of advocates. The subsequent verdict about the safety of X-rays is well known. With regard to obstetric ultrasound, there have been enough reservations as to safety to inspire studies on its deleterious effects. In many cases, critical reviews of studies dismissed results because of methodological flaws. One resilient finding has prevailed - a higherthan-expected incidence of nonright-handedness.?

Safety relating to practice standards is currently seen as the industry's biggest problem. Both internationally and in Australia, practice standards have been found to "fall short" in terms of expert assistance and equipment. One of the major international trials (RADIUS) ${ }^{8}$ that found routine scinning had no benefit on perinatal or maternal outcome was criticized for producing results more attributable to the performance failure of particular operators than a failure in ultrasound per se (Romero 1993:303--307). Although this may demonstrate a problem with the study, it also demonstrates an ongoing problem with regard to operator standards.

Australian standards have also been criticized, with poor performance attributed to a failure in providing necessary training and qualifications (de Crespigny 1995). No prerequisite training requirements exist for operators providing obstetric ultrasound services. Two Australian studies of spina bifida screening" demonstrated that practice standards are inadequate (de Crespigny 1995:31); 40) percent of spina bifida cases were missed at a mid-trimester ultrasound examination. This false negative outcome, combined with the possibility of the converse false positive diagnosis (which can result in abortion of a normal fetus), has prompted calls for rigorous assessment of routine ultrasound "before any confident recommendation that, in practice, it does more good than harm" (Neilson 2000:3)."

Economic studies challenge whether routine use of ultrasound is the most cost-cffective use of limited public health care funding. Based on four million women, at $\$ 200$ per scan, the RADIUS study group calculated the cost of routine scanning at more than one billion dollars per year (Ewigman et al. 1993:827). Oats $(2000: 312)$ argues that government health dollars might better be directed elsewhere, given that the benefits of routine ultrasound are unproven (see also Neilson $2000)$.

\section{Practice Guidelines in Australia}

Australia has a "mixed" health care system. Health services are funded by a national taxpayer-funded health insurance scheme (Medicare) accessible to all citizens and permanent residents as well as from private sources (health insurance and fees paid by individuals) (Belcher 2002:264-266). Medicare provides benefits for treatment by a range of medical practitioners, including primary care practitioners who make referrals to specialists. Benefits are based on a schedule of fees set by the federal government. Practitioners often choose to charge more than the "scheduled fee," in which case the patient meets the remainder.

Most women (about 99 percent) give birth in a hospital setting (including birth centers), either cared for by hospital-employed staff or under the care of a 
private practitioner (obstetrician or general practitioner who takes obstetric cases). About 30 percent of women (virtually all with private health insurance) are under the care of a private obstetrician on a fee-for-service basis (National Perinatal Statistics Unit 1999:Section 2.3). The physician caring for the pregnant woman makes referrals for ultrasound scans and other pregnancy diagnostic tests and screening procedures. The Medicare scheme funds up to three ultrasound scans without further referral of the woman for a second opinion (Sampson 2000:22).

In Australia, as in other affluent countries, the expansion of medical technology proceeds apace, as does the acceptance of the technology in clinical practice. Figures from the Australian Health Insurance Commission for 1997-98 indicate that Medicare funded obstetric ultrasound scans to a total of $\$ 39$ million, out of total expenditure on obstetric-related care of $\$ 96$ million (i.e., 41 percent) (Commonwealth of Australia Senate Committee Report 1999:46). "Surveys within Australia attest to the near-universality of an ultrasound scan at 18-20 weeks, with 97 percent of pregnant women in 1992 having at least one ultrasound scan and 46 percent having two or more (Yates et al. 1995:378). A 1994 survey found that 99.5 percent of respondents reported having at least one ultrasound scan (Commonwealth of Australia Senate Committee Report 1999:45). An earlier scan, at 11-13 weeks, for "nuchal translucency," has been available for several years and is used to establish risk status for a number of chromosomal disorders, including Down syndrome. Increasing numbers of pregnant women are having this scan, despite concerns about its efficacy (Commonwealth of Australia Senate Committee Report, 1999:50-52).

Three professional organizations in Australia have direct involvement with obstetrical services: the Australian Society for Ultrasound in Medicine (ASUM), the Royal College of Obstetricians and Gynaecologists (RANZCOG), and the Royal Australian and New Zealand College of Radiologists (RANZCR). ASUM's statements focus on procedural and safety issues of obstetric ultrasound scanning. RANZCR's policy statement outlines nine "Benefits of Ultrasound in Pregnancy," including anxiety reduction and enhancement of lifestyle changes through promotion of parental bonding. ${ }^{2}$ RANZCOG provides a broader set of guidelines for obstetric practice. RANZCOG's guidelines on "Screening in Pregnancy" contain no recommendations about the use of a routine ultrasound scan at $18-20$ weeks gestation (or at any other time). All statements relate to procedure and protocol only. In the case of the 11-13 week ultrasound scan for nuchal translucency, recommendations on clinical indications as well as "not at risk" cases are provided:

1. Women who are considered to be at increased risk for aneuploidy, based on history (advanced maternal age or previous affected pregnancy) should be offered the choice of either screening (by nuchal translucency scanning [NTS] or second trimester maternal scrum screening), or diagnostic invasive testing (either chorion villius sampling or amniocentesis).

2. Women who are not considered to be at risk should be made aware of the availability of screening tests for DS and other chromosomal abnormalities. [RANZCOG July 2001$]$

The latter recommendation implies that women who are not considered at risk may be able to receive the nuchal translucency scan or other screening tests. This, indeed, is occurring with increasing frequency in the metropolitan areas of Australia, where equipment is readily available. 
The discrepancy between the policy statements of RANZCOG and other professional organizations, with their focus on the clinical indications for ultrasound scans, and the almost universal performance of at least one ultrasound scan on all pregnant women in Australia, raises questions that this study hopes to address. It appears that the perceived benefits of routine ultrasound, by pregnant women and/or the practitioners who care for them, have come to prevail over narrower considerations of clinically defined risk. We turn to the results of our interviews with pregnant women to explore their views about ultrasound scans in more detail.

\section{Methods}

Our data about ultrasound scans are part of a larger qualitative study of pregnant women's conceptions of nutrition and well-being during pregnancy and early motherhood in a regional city of New South Wales (NSW), Australia. Informants were purposively selected to include women of English-speaking background, primiparous delivery, and low risk. This report is based on interviews with 34 women. The mean age of the women interviewed was 26.8 , with a range from 18 to 36 . This sample size is adequate for the analysis, considering the relatively homogeneous cultural group that comprises the sample. Furthermore, we believe that data relating to ultrasound have achieved "theoretical saturation" in that no new themes emerged in later interviews. ${ }^{13}$ In recognition of women's privacy, the analysis is presented using pseudonyms.

Women were recruited with the assistance of general practitioners, who offered obstetric care as part of their practice, and midwives in antenatal clinics of two large public hospitals. ${ }^{14}$ The women were asked to participate in three interviews-ideally, early second trimester, mid-third trimester, and three months postpartum. The study design provides a longitudinal perspective on changes that occur over the duration of the pregnancy and after childbirth. It also allows for crosschecking of information from earlier interviews. In the course of the first and second interviews, women were asked the question: "Could you tell me about any treatments or tests that you have had so far during your pregnancy?" with prompts as appropriate. The third interview, apart from discussion of the birth and postnatal period, allowed for follow-up on sensitive issues regarding tests not dealt with in carlier interviews for ethical reasons (e.g., issues relating to duty of care toward participants). We used open-ended questions and an informal intervicw protocol that encouraged women to speak in their own words about their experiences and opinions. No technical information on diet or other aspects of lifestyle were requested.

The thematic areas of interest in this study arose through an interaction between the researchers and the participants in the study. Ultrasound was not a major concern when we began our study, the primary focus of which was diet and nutrition. However, women themselves often spontancously raised the topic and made such interesting comments about ultrasound that we soon decided to explore the issue more systematically, incorporating a question and prompts into the schedules for the three interviews. ${ }^{15}$ All women in the study underwent the 18-20-week scan, while 27 (79 percent) also had scans at other stages of the pregnancy. In some cases, it is clear that women are commenting on a particular scan; in other cases, it is not possible to distinguish between comments made in relation to other scans 
(usually the nuchal translucency scan) and comments relating to the 18 -20-week scan. Similarly, many of the comments made by women were spontaneous and, as such, we see them as providing an important indication of women's concerns. However, while we endeavor to give some indication of numbers, we do not intend our findings to be statistically representative.

Data analysis was ongoing throughout the study. Two members of the research team coded field notes and transcribed interviews. The QSR Nvivo qualitative data analysis program Version 2 (NVIVO) (QSR International Pty Ltd 2002) was used for coding and further analysis. Coding enabled content analysis to identify commonly recurring themes and themes of theoretical interest (see Table 1).

\section{Patients, Practitioners, and the Government of Pregnancy}

The following sections examine participants' commentaries on ultrasound scans, which we consider in light of the current literature. In particular, we focus on women's comments from a feminist poststructural perspective; that is, we will explore the dominant and marginalized discourses that operate around women's decisions to use ultrasound during pregnancy while acknowledging women's own experiences: their prior understandings about the world as well as their embodied knowledge.

Drawing on a Foucauldian concept of discourse analysis, where discourse can be thought of as "practices that systematically form the objects of which they speak" (Foucault 1972:49), we will examine the discursive constructs underpinning women's involvement with obstetric scans. In particular, we aim to explore the "techniques of power" through the regulatory mechanisms of "biopolitics" (Foucault 1990:141). This focus allows us to identify processes of the subjection of individuals in comparison with a statistical norm and the production of risk discourse, both of which are pivotal to strategies of normalization (Lupton 1999:61). Such strategies enable a method of government whereby populations and individuals self-regulate by aspiring to expert-defined norm, referred to by Foucault as a process of "governmentality."

The idea of the government of pregnancy by a process of individualized risk management is supported by an assemblage of literature (Dean 1999; Ruhl 1999; Weir 1996) and is described by Weir as "a kind of government by security" (p. 379). Ruhl argues that responsibility for fetal health (in contrast to maternal health)

Table 1

Ultrasound scan history of 34 study women.

\begin{tabular}{lrr}
\hline & $N$ & $\%$ \\
\hline 18-20 Week Scan & 34 & 100 \\
NT Scan & 14 & 41 \\
> Scan & 27 & 79 \\
2 Scans & 14 & 41 \\
3 Scans & 9 & 26 \\
4 Scans & 3 & 9 \\
5 Scans & 1 & 3
\end{tabular}


has been increasingly attributed to individuals, including individual control $/ \mathrm{man}$ agement of risk factors. Fetal health is dependent on the regulation of dict (including alcohol and tobacco intake), exercise, and compliance with prenatal care recommendations, among other factors. As Ruhl points out, the effectiveness of the "individualised risk" model of pregnancy is that rather than being punitive or simply misogynistic, it enlists the efforts of pregnant women in their own government.

Our research is based on a concept of the body that is both socially constructed and material at the same time (Butler 1993). While bodies "only appear, only endure, only live within the productive constraints of certain highly gendered regulatory systems" (Butler 1993:x), embodied experiences are no less real than any material or political reality. One of the effects on women with which we are concerned is the way technology becomes a part of women's embodied knowledge, in a phenomenological sense. Cultural meanings are intrinsic to embodied experience and experiential knowledge is not fixed. The body, and how it is experienced, cpitomizes flux (Frank 1991:40).

Historically, women have been situated as "embodied" subjects, within a dualism that privileges the mind (the masculine pole) and considers the body (the feminine pole) as biological, material, fixed and pre-cultural. Women's "embodiment" has been used to conlirm their inferiority to men. Recent theorizing, as Csordas (1994:9) points out, is heir to its Cartesian legacy in that it continues to situate the body as a representation by privileging the mind/subject/culture set. Scholars have failed to consider "lived experience" in a phenomenological sense. ${ }^{10}$ Where embodiment has been considered, it has been argued that we tend not to be aware of our bodies unless experiencing hunger or pain, or other pressing bodily needs (Leder 1990). Lupton, however, points out that such assumptions can be criticized for the presentation of embodiment as lacking gender. Women are acutely aware of the attention of others toward their appearance, especially during pregnancy (1999:60).

We follow Browner and Press's definition of embodied knowledge as knowledge that is subjectively "derived from a woman's perceptions of her body and its natural processes as these change throughout her pregnancy course" (Browner and Press 1997:133). Our focus on discourse analysis and women's embodied and experiential knowledge will allow us to compare the degrees to which women's ways of knowing are shaped by biomedical authority and techno-medicine during this time. It allows us to examine the degree to which, and the reasons why, women have embraced ultrasound and to theorize the impact of this acceptance.

\section{Practitioner-Patient Communication about Ultrasound Scans}

Our study focuses only on pregnant women's comments regarding their health practitioners' advice. Exactly how health practitioners present the risks and benefits of ultrasound has not been directly investigated. Women reported that practitioners present the 18-20-week ultrasound as a routine part of pregnancy procedure: "She said it was routinely done." A number of women (particularly those receiving care through the public system) stated they were told to have the test: "They basically said 'You need to have this scan to see whether the baby's doing what it should be doing and if it's positioned properly.' ", 
The information that practitioners reportedly offered about the purpose of the scan was sparse. It ranged from none, in many cases, to explanations (in a small number of cases), that the scan was to check size, positioning and potential problems. Statistical possibilities were discussed: "She told me what the possibilities are and what the chances are of that happening, and that in my case it was highly unlikely that any of that would happen" (Nadine). In two cases, women who expressed reservations about having a scan were encouraged to do so on the basis that surgery could be performed in utero.

Women reported that their practitioners provided greater information in relation to the first trimester nuchal translucency (NT) scan discussed above. The majority of women who seriously considered having the NT scan reported their practitioners discussing what the test was for, ${ }^{17}$ the reasons for the timing of the test, and who ought to consider taking the test. They also were informed how the information on risk status was obtained: "They ... measure the fold of skin on the back of the baby's neck" (Annie). Women who were told they had genetic risk factors reported that their practitioners recommended the test, as did women with other pregnancy concerns, ${ }^{18}$ and women in higher age categories. Assessment of risk because of higher age apparently did not follow the RANZCOG recommendations that prenatal diagnosis should be available to all women of 37 years and over ( 35 in NSW).

Our sample included six women in the "grey area," between 30 and 35, all of whom opted to have the NT scan, following discussions with practitioners, because of their age. As one woman put it:

It was sort of like, if you're going to be 35 and over we really need to check these things early, and if you were like 32 and under then you're a lot lower risk, but because you're in this little grey area of being between 32 and 35, then my GP actually said "you should" you know "maybe have these tests anyway that they would expect to do if you were a little bit older."

Some women outside the "higher risk" groups stated that their practitioners discussed the availability of the test but did not actively recommend it. This is also in accordance with RANZCOG guidelines, which recommend that women who are not considered to be at increased risk should be made aware of the screening tests (RANZCOG 2001). This was not the case for one woman receiving private care who stated her obstetrician presented the scan as a "routine" procedure: "The twelve week [NT] scan was recommended by my obstetrician. He suggests all his patients have it because it's noninvasive" (Donna).

The reported presentation of the 18-20-week scan as routine and nonvoluntary, along with the lack of information accompanying the referral, suggest that medical practitioners consider the scan as an unproblematic and necessary aspect of pregnancy care for the "low-risk" women in our study. Mitchell and Georges (1997), in a cross-cultural study ${ }^{19}$ examining North American and Greek understandings of the "cyborg fetus," 20 similarly comment that physicians and sonographers (ultrasound operators) consider ultrasound as a necessary, passive, and neutral technology. ${ }^{21}$ The focus of practitioners on rectification of potential problems as opposed to interventionist aspects of the scan could be attributed to a concern of individual practitioners to avoid causing undue anxiety. This, however, does not alter the fact that public health benefits of screening rely not on therapeutic interventions 
but on abortion to achieve better live-birth statistics. Nor does this approach provide women with the opportunity of giving informed thought to possible outcomes.

\section{Understandings of Screening and Its Consequences}

Previous studies investigating women's understandings of the consequences of screening procedures have found that participants have failed to adequately consider termination of pregnancy. Press and Browner (1997:986) concluded that women had subsumed the maternal serum alpha-fetoprotein (MSAFP) screening test under the rubric of prenatal care. The technology thus became imbued with the meanings and perceived advantages that pregnant women associated with that care. These included the provision of information, emotional reassurance, and prevention of fetal and maternal harm. This, they argue, is problematic, given that screening is not about the care of the pregnancy, but the prevention of the abnormal full-term birth via abortion. ${ }^{22}$

Mitchell and Georges (1997:394) similarly found that Canadian women considered routine ultrasound "primarily as a means of "doing what's best for baby." They theorized that ultrasound in Canada is "firmly embedded in an individualizing ideology of risk and maternal responsibility." They demonstrated that women failed to adequately consider the next logical step within a neoliberal society: termination of pregnancies in the interest of public health.

Research investigating prenatal testing (including ultrasound and maternal serum) and diagnostic tests (amniocentisis and CVS) among Cambodian, Lao, and Vietnamese women in Australia (Liamputtong and Watson 2002) also found that reassurance was the clearest rcason for women to agree to prenatal testing. Citing Farrant (1985) and Green et al. (1993), Liamputtong and Watson argue that "the main reason that women agree to screening is not to detect abnormality in their baby, but to reassure them that there is no abnormality" (2002:310).

For the women in our study, a marked contrast emerged in the understandings of the screening implications of the 18-20-week scan compared to the carlier NT scan. Generally, the issue of termination was well considered among those women who underwent NT screening. Almost all the women who had taken or declined a NT scan demonstrated a clear understanding that this procedure screens ${ }^{23}$ for the genetic condition of Down syndrome, but only one mentioned screening for other chromosomal defects. A number of women indicated that they were prepared to terminate their pregnancies should the test (and subsequent testing) indicate the likelihood of the condition. Others stated that their reason for undertaking the test was to gain knowledge in order to prepare themselves. Three women opted not to take the test because they knew they would not terminate their pregnancies, while others stated they had yet to make up their minds or else had not thought too much about what they would do should a problem be detected.

The recognition by our study participants of pregnancy termination as a possible outcome after the NT scan contrasts with Press and Browner's findings (1997:986) relating to the use of prenatal screening. Our participants largely considered the consequences of this test. The different findings are likely attributable to the depth of information about the NT test provided by practitioners. Other studies suggest that particular cultural values may reinforce the understanding of screcning as a precursor to possible termination, as was the case with the Greek 
women studied by Georges (1996). Our interpretation of differences in findings derives from various observations outlined in Georges (1996) and Mitchell and Georges (1997), including the signifying of fetal personhood by fetal physical normality (for Greek women); the diagnostic presentation of the test in the Greek setting; and the immersion of women in a public discourse of abortion that emphasizes the greater cultural good of the community over maternal choice and fetal rights.

Among the women in our study, this understanding of possible screening outcomes was largely absent in relation to the 18-20-week test. Women who had undergone the NT test with the stated purpose of detecting Down syndrome made much less specific comments about the purposes of the 18-20-week scan: "I have to go back when I'm eighteen, nineteen weeks to get another one done. Just to make sure everything is going all right" (Sally), and "I'm booked in for the one you're supposed to have between 18 and 22 weeks to see how well the baby's doing" (Annic). Such comments are in keeping with those of women who had not undergone NT testing, who also tended to mention the general benefits of the 18-20week U.S. scan, as described above. Only four of the 34 women demonstrated a clear understanding of the screening purposes of the 18-20-week test, which is to identify abnormalities. This may be partly attributed to the participants' low-risk status, which was a critcrion for joining the study. Women in our study, as elsewhere (Liamputtong and Watson 2002:309; Mitchell and Georges 1997:394; Press and Browner 1997:986) appear to perceive the test as a responsible action within their eontrol that produces a better pregnancy outcome.

Like the women interviewed in Press and Browner's study (1997:986), a number of our study participants did not initially comment on the scan at all, simply adding it to the list of other prenatal tests: "just the blood test and the scan, that was all." Many others normalized the scan, with the word "routine" occurring frequently: "So I had the routine two blood tests and the routine two ultrasounds." Generally, the scan was considered a responsible thing to do: "I think that's why you, it's more important to look after it while it's there, it's your responsibility" (Angela).

How an ultrasound scan could alter pregnancy outcome other than termination, however, was largely unarticulated. In many cases, inadequate understanding of the purpose of the test suggest that women could well be faced with decisions they would have preferred to avoid, compounded by social pressures that make a terminated pregnancy the responsible course of action (Lupton 1999:69). The normalization of ultrasound in antenatal care, by both women and practitioners, has the potential to obfuscate the ethical issues of abortion and disability rights and other concerns such as the economics of health care, pregnant women's informed consent, and the right to decline tests.

\section{Questions of Safety}

The salety of the 18-20-week ultrasound was not an issue that most women reported discussing with their practitioners. Nadine, a particularly well-informed participant with an "alternative" health background, discussed both cost and safety with her practitioner during her pregnancy and reportedly was told: "The government prefer you to only have one scan, for financial not safety reasons." When 
Nadine probed further on the safety issue, the response reportedly was: "She told me that it was, well, that it hadn't been proven unsafe yet."

This sort of statement that women report from practitioners or pregnancy books is in line with the Statement on the Safety of Ultrasound in Grey Scale Imaging in Obstetrics (reaffirmed February 2000), of the Australasian Society for Ultrasound in Medicine (1999) ${ }^{24}$ :

Grey scale imaging by transcutaneous, transvaginal and endoscopic routes is a well-established procedure in diagnostic medicine. To date, the results of followup studies on patients and children who had been examined before birth have not demonstrated a causal link between adverse health effects and ultrasound exposure. ${ }^{25}$

In our interviews, the issue of safety generally did not emerge unless prompted. When discussed, largely in the postnatal interviews, a small number of women stated they did not believe there to be any risks associated with ultrasound. A few more discussed safety in terms of lack of proof that ultrasound can have a detrimental effect on pregnancy outcome. Natalie stated that the lack of proof meant that she was "not worried about that at all." A small number of women stated that safety remained a concern and that due to safety concerns, they wished to keep scans to a minimum: "In my own mind I'd prefer to have less scans anyway" (Nadine). In accordance with this wish, Nadine chose not to participate in a study requiring her to have five scans. ${ }^{26}$ Gina said she kept her scans to a minimum "and that ended up being three." Similarly, two women stated they were aware of recent research "that's just come out saying ultrasound [can] potentially cause some defects." Both stated they believed the possible preventative measures of a scan outweighed any negative effects, and it was a matter of weighing up the risk: "I suppose there is a place for everything, and if there's a risk of there being a problem with your baby and you want to know then I suppose you sort of take your chance" (Helen).

Safety issues came up more frequently in relation to NT scans, in our interviews. This is probably because of their status as a precursor or alternative to more "intrusive" tests: amniocentesis and Chorionic Villus (CV) sampling. Safety issues in this context related to an increased risk of miscarriage associated with amniocentesis and CV sampling, and, conversely, a conceptualization of ultrasound as less risky, rather than consideration of other possibilities, including damage to the baby or mother.

Safety in relation to the fallibility of scientific evidence was also considered. Many women who had or considered a NT scan spoke of the inconclusiveness of NT scan results: "I wouldn't have done anything anyway. I mean they' re not conclusive" (Donna). ${ }^{27}$ The majority of women who took the NT scan had been advised of this lack of certainty by their medical practitioners. Two others had personal knowledge of incidents where incorrect information had been provided. It is interesting that women's concerns about fallibility all relate to scanning that they were told was for specific indications. Only Erin articulated concerns as to the fallibility of the 18-20-week scan, where the purpose of the scan was presented as less specific. In this instance, the comment was based on personal knowledge of incorrect scan results: 
A couple of people that I've worked with, they've had scans, and one of them had a scan and was told their baby didn't have a stomach and then as soon as their baby was born they'd have to go to theatre. And it wouldn't be able to feed. And it was born perfectly healthy. And you know, he just said "We were talking about terminating, we were thinking what kind of life is this for a baby," you know, and he said "and it was fine." So like you hear that side of the story, too, it's not 100 percent accurate.

A small number of women also commented indirectly about fallibility in relation to not having access to photos or video recordings of their scans, for what had been explained as legal reasons:

And the video, they don't do it now for legal reasons . . it's been brought out now about the photos as well, just in public hospitals. If Medicare covers it then they don't do it, but that's because if you find, you know if your baby is born with some deformity and they didn't pick up on it, then you can sue them for not picking up anything. [Heather]

This practice is in line with warnings by ASUM that: "Providers should be aware of the medico-legal implications if a missed lesion is demonstrated on the image(s) given to the parent(s)."28

The previously discussed notion that the 18-20-week scan could somehow produce a better pregnancy outcome was augmented by our participants' trust in biomedical technology. This trust can be inferred from the lack of concern regarding the safety aspects of the technology. Among those women who did conceptualize a potential risk, the risk of rejecting the scan and thus foregoing a (perceived) chance to help the baby, or the chance to terminate the pregnancy, outweighed the potential harm to mother or baby. Nevertheless, despite women's trust in the benefits of the test, trust in results was less conclusive. This reinforces the notion that women do not expect to have to make decisions about termination and presents a further dilemma for parents who do.

\section{Ultrasound as Embodied Practice in Pregnancy: Anxiety and Reassurance}

While pregnant women historically and cross-culturally may experience anxiety about their pregnancies, the production of risk discourse particularly about pregnancy has increased the anxiety levels and concerns expericnced by pregnant women. Only two women stated they did not experience any anxiety in relation to scanning. Anxiety in relation to the 18-20-week scan was articulated more in terms of lingering, ever-present doubts about the abilities of women's bodies to cope with a pregnancy. Natalie, who said she felt physically sick due to her nervousness at having a scan, spoke of her concerns that something could be wrong despite the fact she was fit and healthy: "Like I think I'm young, I'm healthy, I'm fit, everything's just got to be fine, but at the same time, you know, it's just it's just in the back of your mind."

Women also expressed anxiety in terms of having to make a decision as to whether to terminate the pregnancy and in relation to the fallibility of NT scan results. Where termination was not considered an option for reasons of personal values, continuing the pregnancy with knowledge of an anomaly prevented two women from having the NT scan. 
Generally, women experienced the greatest anxiety in relation to their first scan. Leah discussed the combined anxiety and relief posed by the results of her NT test:

It was just a horrible thing to actually deal with, that the relief afterwards was quite remarkable. . . I said to my husband, I wasn't aware of how tense I was up until then and he was a little bit the same as well, becaluse there was all this unspoken stuff that you avoided. What if? What if it's not right?

It was common for expressions of anxiety to be linked with reassurance following scan results. Women spoke of "normal" and "average" babies: "It was really good, the baby was average size." Indeed, testing was presented more in terms of helping relieve apprehension about pregnancy and provide peace of mind. Iona described the NT scan as good, not in terms of relieving anxiety about Down syndrome, but "because that early on I was scared about losing the baby." The scan provided reassurance by confirming the normality of the baby.

Julia, a health professional, captures the quandary faced by women as to how best to avert anxiety in the presence of the technology and the accompanying discourses of risk. Julia expressed concerns about the safety of the scan, having to make a decision about termination if indicated, and not wanting the anxiety pro ducing knowledge that her baby may have a disability. She also articulated concerns about transferring her anxiety to her child:

\section{Yeah, so I've decided, during this pregnancy, I wouldn't have it because I don't want to know because I don't want to be anxious. Because I do think that if I'm anxious ... if I feel stressed then the baby gets this big dose of stress hormones and I can't help but wondering again if in their post-birth life that they're not anx- ious as well.}

Julia, nevertheless, opted to have a scan. When asked why she had relented, she responded, "Partly I think to, I don't know, partly to I guess eliminate that anxiety."

Antenatal technologies are a site where epidemiologically based notions of risk are individualized. For cxample, in relation to nuchal translucency scanning, comments such as Leah's were common: "So combining the results of the blood test and the scan, my age, they then count the odds of genetic disorders and Down syndrome. And those odds were quite long, so I was quite pleased with that."

In an Australian study on first-time motherhood involving 25 women, Lupton demonstrated how women's concepts of risk differ from expert concepts, which rely on statistical concepts of probability. "Women draw not only from expert knowledges but from their own experiences, their knowledge of other"s experiences and lay concepts of risk which circulate in their cultural context" (Lupton 1999:76).

This account of dealing with risk was very much evident in our study participants' choices about NT scans and dealing with negative outcomes. The benefits of the screening purposes of ultrasound scans go far beyond the intended medical "benefits" of termination, surgical intervention, and saving the public health dollar. Where termination was deemed a possible outcome of the scan, only one woman discussed her reasons in terms of eugenics, stating she believed it cruel to bring a disabled child into the world. All couched their comments in terms of not being able to cope or losing control. Sally felt her age and inexperience impacted 
on her ability to cope: "I probably would have terminated, because like I've watched Henry, my little cousin grow up, and my auntie go through it all and she says she can't handle him any more. And like she's 35, 36, and like I don't think I'd be able to handle it myself."

Decisions about termination/undertaking the test were also linked to the women's experiences in conceiving and intentions regarding family size. Annie's intention to terminate should there be an adverse result was based on a decision between herself and her husband to have only one child. That being the case, it was important to them that the child be "normal." Gina, who conceived via in vitro fertilization and Tanya, who had experienced a miscarriage, both opted not to have a NT scan because they would not terminate the pregnancies anyway.

Our findings, like Press and Browner's (1997:986), Georges's (1996), and Mitchell and Georges's (1997), suggest that scanning in pregnancy both reinforces and relieves preexisting anxieties. The preexisting anxieties relate, in part, to ways in which antenatal technologies enforce the notion of the hazards of pregnancy, situating all pregnancics as "at risk" and making the "no-risk" pregnancy obsolete (Lupton 1999:68). From a Foucauldian perspective, this interplay can be understood in terms of the double dynamics of power, that is the power that weighs on us, as well as that which induces pleasure (and knowledge) (Foucault 1980:119, 1996:165). Indeed, the knowledge and pleasure induced by ultrasound was discussed at length by the women in our study.

\section{Seeing for Knowledge}

For the women in our study, "normality" was confirmed and reassurance afforded by the ultrasound operator's interpretations of fetal images. Despite questioning the fallibility of scientific evidence about specific indications of aneuploidy or the sex of the baby, women did not question practice standards, neither did they question the sonographer's more general interpretations of images: "I've got an appointment with her [GP] in a couple of weeks to get the results of the ultrasound, which the ultrasound technician said looked good anyway. So I'm not nervous about that" (Iona).

Seeing the baby also reassured some of our women about the changes taking place in their bodies: "That's when it sort of makes it real and you think, oh that's inside of me" (Hanna). Cara also described how "seeing the baby" gave her knowledge her pregnancy was real:

I think after I had my first scan it didn't . . like I knew I was pregnant, but it didn't really hit me until I saw something live and physically happening inside me. And from then I've been able to accept much more about the changes and I've noticed weekly, you know, l've just felt weekly that, yes, I can feel my stomach changing.

Such comments indicate, as feminist theorists have argued, the dislocation of experiential knowledge. As with Greek and Canadian women, the women in our study "enjoyed and [came] to rely on a technologically mediated introduction to [their] pregnanclies]" (Mitchell and Georges (1997:383). The experience of "seeing" "demoteld] bodily experience to a second order significance" (Georges 1996:164). 
In a generation brought up in an interpersonal world dominated so thoroughly by visual media such as TV and home videos, it is perhaps not surprising that the visual aspects of ultrasound provided women with a sense of reality about their pregnancies. Also, when combined with the authoritative voice of practitioners, the images themselves gave reassurance of the "normality" of pregnancies. Georges succinctly describes the power of fetal ultrasound, as deriving from:

its unic|uc positioning at the intersection of populat and scientific lechnologies of the visual and with eodes and conventions of representing the "real" that are embedded in these technologies... In ultrasonography the visual and the scientific--two highly significant components of the modern in Western culture-are deliberately joined together. [Georges 1996: 1.58]"

\section{Secing for Pleasure}

The pleasure of "seeing the baby" was the issue most often and clcarly discussed (without prompting). Many of the women mentioned their excitement/interest at seeing the fetus: in fact, two women said seeing the baby was the primary reason for additional tests: "Our doctor offered us the one at 11 weeks, that nuchal translucency scan. To be honest we were just excited to see the baby, so we said 'Yeah, we'll have that one, too'" (Erin).

Some women who had multiple scans deemed the 18-20-week scan the most pleasurable. There was a greater possibility of distinguishing "little legs and litte arms" (Cara). It was also the first opportunity to photograph and videotape the baby.

The pleasure of seeing the baby even affected Julia, despite all her anxieties at having a scan:

So that was a bit why I was reluctant to have it done, but as it was, it wals a very positive experience becaluse they said: "Oh, it's fine." And it wals really lun to sec this litte baby on the sereen and see it moving around, and that was really good. So, despite my initial anxieties it was good, it was a good experience, and f'd actually say 10 others it was a really nice thing to have done.

The imaging of the fetus also provided an opportunity for the woman and significant others to construct a persona for the baby. Renee discussed following the development of the foetus, comparing her baby to the images she had seen in books: "So it was $4.3 \mathrm{~cm}$ long, just sort of looked like a peanut. . . I have been reading up in the book about what size it will be now and when it is getling its hands."

Iona said that finding out her baby was a girl and giving it a name, made everything seem more real: "And the second |scan| was good, because we found out it is a girl. So it has kind of made it a bit more real, just the baby and immortal [sic] person. It is good, we have a name and we have talked to it, with its name."30

Corrina discussed the family resemblance of the fetus: "Mum was just fascinated, never seen anything like it and she was just really fascinated. I remember her saying, 'It looks like John.' I don't know how you could tell. But anyway, she was really excited, she was good to bring along because she got really excited." 
The scanning procedure also allowed partners to see the baby, and was presented in some cases as part of their socialization process as a dad. "Part of it was that it was an opportunity for Brad, my husband, to see the baby" (Julia).

Such pleasures are akin to those of the Canadian women of Mitchell and Georges (1997) study, who similarly attributed personality and agency to the fetus and contrast to Greek women, who did not recognize fetal autonomy or subjective characteristics (Mitchell and Georges 1997:389). The double dynamics accompanying the personal pleasure of fetal personification includes, at a societal level, a potential conflict, with its many political implications, between the rights of the mother and of the fetus (see Zechmeister 2001:393-395).

\section{Conclusion}

The findings of this study clearly indicate that ultrasound scanning has become an integral part of the clinical care of pregnant women. Nevertheless, women's understandings of the purposes of the 18-20-week scan are poor. Although we did not have direct access to women's consultations with practitioners, women's reports suggest that practitioners, responding to both consumer demand and legal concerns, are attempting to impart complex and often inconclusive information about risks and benefits of ultrasound screening within the time constraints of a busy clinic or private practice. These circumstances give rise to partial understandings of the purposes of screening and the broader outcomes. These understandings inevitably inform women's readiness to undertake ultrasound scans.

The ultrasound phenomenon, however, does not derive from the authority of the practitioner, but rather from its location at the convergence of powerful representations of reality: the visual and scientific. It has been embraced by women themselves for a variety of reasons, including the pleasures of seeing the baby while still in utero, and the sense that ultrasound scans are part of the responsible management of pregnancy that enhance the chances of a positive outcome. These reasons are linked to the importance of having control over the pregnancy (and if necessary, foreknowledge of an adverse outcome). Those women who chose to minimize screening because they would not terminate the pregnancy considered control more in terms of their embodied experiences: controlling their anxiety levels and enjoying their pregnancies.

As Foucault maintains, power is at the same time both repressive and productive (1980:119), and there is a price to be paid for the knowledge and pleasure experienced by our study participants. The increasing use of the technology by practitioners has augmented the role of scientific biomedicine in the governance of pregnancy. While professional authority might previously have been based on the experience and standing of the individual practitioner, it is now augmented by techno-scientific procedures that provide even less space for a "layperson's perspective." Similarly, women's embodied knowledge becomes devalued, taking second place to the masculine visual knowledge of surveillance (Petechsky 1987; Zechmeister 2001:392). This is the case even where the data are conflicting and inconclusive, as in the case of ultrasound scanning, so that practitioners often have recourse to citing the negative evidence, such as the often-repeated assertion, "It has not been proven unsafe yet." 
There has been a transformation of antenatal care of the mother by the addition of the technical surveillance of the fetus. This marks a contemporary extension of the medicalization of the family, identified by Foucault, as well as revealing the gendered nature of the process. Foucault argued that since the 18 th century, private responsibility for health maintenance has been "articulated onto a collective system of hygiene and scientific technique of cure made available to individual and family demand by a professional corps of doctors, qualified and, as it were, recommended by the state" (Foucault 1984:281). In the case of obstetric scans, the scope of responsibility for family health has been extended to include the fetus. Responsibility rests firmly with the pregnant woman, whose custodianship of the fetus must meet the standards of medically defined risk management.

The use of this antenatal technology has the power to transform women's embodied experience of pregnancy. The fetus, once "seen," becomes a separate entity for which the woman is responsible. She becomes morally (and perhaps legally) culpable for damage that may ensue from a failure to exercise microregimens of care (such as healthy diet, appropriate exercise, and medically mandated antenatal screens and tests). The pregnant woman is exhorted to be a responsible agent acting in the interests of the fetus, while also subject to the rationality of expert medical knowledge. The tensions and anxieties around screening and other aspects of antenatal care expressed by women in our study are testimony to the way in which pregnancy has been discursively constructed as a risky domain of gendered experience in contemporary Australian society.

\section{Notes}

Acknowledgments. The study from which this paper is derived was funded by an Australian Research Council Small Grant and a University of Newcastle Research Scholarship. The authors wish to thank all participants in the study as well as the health professionals who assisted with recruitment. We are also grateful to the Qualitative Research Laboratory at the Centre for Clinical Epidemiology and Biostatistics, University of Newcastle, for assistance with transcriptions.

1. It has also become common in some developing countries (India, for example), primarily for purposes of sexing babies (see Gill 1998; Sheth and Malpani 1997). Discussion of these issues is beyond the scope of this article.

2. To our knowledge, there are no systematic comparative surveys as to the prevalence of scans in different countries. Our information is drawn from a variety of different sources (for Germany, O'Brien et al. 1993; for Norway, Saetnan 1996; for Australia, Yates et al. 1995; for Greece, Georges 1996: 157; for the United States, Ancrican College of Obstetrics and Gynecologists 1997).

3. A recent Norweigan study was based on scans of embryos less than $10 \mathrm{~mm}$ in size (Blaas et al. 1998).

4. 4-D in this context refers to dynamic or motion 3-D (Woo 2001).

5. This is a simplified account of a complex set of historical processes. For more detail, refer to Wajeman (1994).

6. Anecdotal evidence suggests that an ultrasound determination of due date can be critical information in the decision making about to attempt the costly process of saving the life of a very premature baby (Sydney Morning Herald, Good Weekend, August 11, 2001).

7. The Cochrane collaboration concluded that the Norwegian findings may be a chance finding, alternatively if substantiated the finding "could imply that the effect of 
diagnostic ultrasound on the developing brain may alter developmental pathways" (Neilson 2000:6).

8. The Routine Antenatal Diagnostic Imaging with Ultrasound (Radius) trial, funded by the National Institute of Child Health and Human Development, was conducted in the early 1990s in the United States (Ewigman et al. 1993). The randomized trial involved 15,151 pregnant women and sought to determine whether ultrasound screening decreased the frequency of adverse perinatal outcomes.

9. The detection rate for spina bifida is argued to be an excellent indicator of the quality of ultrasound services due to its relative commonness ( 0.1 percent of pregnancies) and ability to be accurately diagnosed (de Crespigny 1995:31).

10. See Getz and Kirkengen (2003), for an account of the potential (and documented) moral, emotional, and physical consequences stemming from prenatal testing and the counseling of women on the basis of insufficient data.

11. This figure does not include scans performed in public hospitals for antenatal clinic patients; thus it is the lowest estimate of cost.

12. RANZCR (1998). See www.ranzcr.edu.au/open/pol3_1.htm, June 1998.

13. Previous qualitative research documenting health beliefs in other countrics has found "theoretical saturation" can be achieved at 20 interviews (Helitzer-Allen and Kendall 1992; Martin 1987).

14. Women attending early pregnancy antenatal consultations (normally within the first trimester or carly in the second trimester) were given information and consent sheets and asked to return them via a reply paid envelope should they wish to participate. Demographic details (i.e., parity, risk, class status) were assessed and appropriate women invited to participate lurther.

15. Thus, the carly interviews we carried out had less information on this topic, but we were able to follow up in the second interview with these women.

16. Csordas argues that the dominance of semiotics over phenomenology is based on a representialist theory of language that polarizes language and experience. So, the claim that "you cannot study experience, because all experience is mediated by language," invites the counter-argument "that language gives access to a world of experience in so far as experience comes to, or is brought to, language." See Csordas (1994) for a more detailed argument.

17. Women spoke only of detecting Down syndrome not other genetic disorders.

18. Stacy had been taking medication that increased the risk of deformity, and Annic reported blecding in carly pregnancy. It should be noted that these are the women's interpretations of medical advice.

19. This involved 158 pregnant women.

20. The cyborg, as described by Donna Haraway (1991) is a "co-construction" of humaus and none humans. In the case of the cyborg fetus, this constitutes the coupling of human and machine to achieve "true" (accurate, scientific) knowledge of the fetus.

21. The threat of medical malpractice suits could also influence GPs' recommendations of ultrasound; however, the relative lack of information accompanying referral suggests that this was not the casc with our study participants' practitioners.

22. Although we agree with Press and Browner (1997) and Mitchell and Georges (1997) that pregnancy screening in a neoliberal society is about termination of pregnancies, this is not neccssarily the view of the stakcholders involved, for example, pregnant women themselves, medical practitioners, and disability rights activists. The point of view of stakeholders, other than pregnant women, goes beyond the scope of this article. For discussions of physicians' perspectives, see Novaes (2000) and Strauss (2002). A good discussion from a disability rights perspective can be found at the "Our Bodies, Ourselves" website http://www.ourbodiesourselves.org/gendis.htm. 
23. Women are deemed to understand the screening aspects of ultrasound if they demonstrate a clear understanding of the medical purpose of the test and thoughtful consideration of an outcome.

24. See www.medeserv.com.au/asum/open/policies $08 / \mathrm{h} 1 \mathrm{~m}$.

25. The statement then proceeds to a brief recommendation regarding the "prudent" levels of "acoustic output" and "dwell time" for the procedure. The definition of what is an "adverse health effect" is not unambiguous. The one large-scale randomized Australian study undertaken to datte (the "Raine" study) compared children of mothers who had hatd one ultrasound versus five. It was claimed there were "no deleterious effects on the children whatsoever, apart from a one per cent shift in the birth weigh |sic| eurve to the left in babies that had frequent ultrasounds," an effect that disappeared by one year of age (cited in Commonwealth of Australia Senatc Committee 1999:50). As indicated in an earlier section of the article, lindings of nonright-handedness ol children exposed to ultrasound in utero, albeit in studies of smaller scale, are quite consistent internationally. But these effects, apparently, or what they might indicate, do not count as "deleterious."

26. Another of our study participants did choose to participate.

27. The issue ol probabilities and fallibility also came up in relation to sexing the baby: "Well, the ultrasound said 95 percent that we were having a boy."

28. Sec ASUM 1999:Statement B3.

29. See also Zechmeister (2001), who similarly argues that the impact of ultrasound and the positive attitude afforded the technology by women is owed, in part, to the combined effects of the importance of the "visual" in Westen culture and the authority alforded medical cxperts.

30. But she still refers to the fetus as "it."

\section{Riherhinchs Citho}

American College of Obstetrics and Gynecologists

1997 Routine Ultrasound in Low-Risk Pregnancy. ACOG Practice Patterns: EvidenceBased Guidelines for Clinical Issues. Intemational Journal of Gynaecology and Obstetrics 59(3):273-278.

Australasian Socicly for Ultrasound in Medicine ( $\triangle S L M)$

1999 hitp://www.asum.com.au/open/home.htm.

Bekcher, I Ielen

2002 Power, Politics and Health Care. In Second Opinion: An Introduction to Health Sociology, 2nd ed. John Germov, ed. Pp. 257-282. Melbourne: Oxford University Press.

Blats, H. G., S. H. Fik-Nes, S. Berg, and H. Torp

1998 In-Vivo Three Dimensional Ultrasound Reconstructions of Embryos and Iarly Fetuses. Lancet 352:9135.

Browner, Carol II., and Nancy Press

1997 The Production of Authoritative Knowledge in American Prenatal Care. Medical

Anthropology Quarterly 10(2): 14 I-156.

Butler, Judith

1993 Bodies that Matter: On the Discursive Limits of Sex. New York: Routledge.

Commonwealth of Australian Senate Committee Report

1999 Rocking the Cradle: A Report into Childbirth Procedures. Available online: www.aph.gov.au/senate/committed/clac_ctle/child_birth/[June 17, 2002].

Csordas, Thomas J.

1994 Introduction: The Body as Representation and Being-in-lhe-World. In Limbodiment and Experience: The Existential Ground of Culture and Self. Thomats J. Csordals, ed. Pp. 1 26. Cambridge: Cambridge University Press. 
Davis-Floyd, Robbie E., and Carolyn F. Sargent

1997 Introduction. In Childbirth and Authoritative Knowledge: Cross-Cultural Perspectives. Robbie E. Davis-Floyd and Carolyn F. Sargent, eds. Pp. 1-54. Berkeley: University of California Press.

Dean, Mitchell

1999 Risk, Calculable and Incalculable. In Risk and Sociocultural Theory: New Directions and Perspectives. Deborah Lupton, ed. Pp. 131-159. Cambridge: Cambridge University Press.

de Crespigny Lachlan C. H.

1995 Standards of Mid-Trimester Obstetric Ultrasound in Australia. The Medical Journal of Australia 163:31-32.

Enkin, Murray, Marc J. N. C. Keirse, Mary Renfrew, and James Neilson

1995 A Guide to Effective Care in Pregnancy and Childbirth. Oxford: Oxford University Press.

Ewigman, Bernard G., James P. Crane, Frederic D. Frigoletto, Michacl L. LeFevre,

Raymond P. Bain, and Donald McNellis

1993 Effect of Prenatal Ultrasound Screening on Perinatal Outcome. English Journal of Medicine 329(12): 1474-1478.

Farrant, Wendy

1985 "Who's for Amniocentesis?" The Politics of Prenatal Screening. In The Sexual Politics of Reproduction. Hilary Homans, ed. Pp. 96-122. London: Gower.

Foucault, Michel

1972 The Archaeology of Knowledge. London: Tavistock.

1980 Truth and Power. In Power/Knowledge: Selected Interviews and Other Writings, 1972-1977. Colin Gordon, ed. Pp. 109-133. Brighton, Sussex: Harvester Press.

1984 The Politics of Health in the Eighteenth Century. In The Foucault Reader. Paul Rabinow, ed. Pp. 273-289. New York: Pantheon Books.

1990 The History of Sexuality. Vol. I: An Introduction. New York: Vintage Books.

Firestone, Shulamith

1970 The Dialectic of Sex: The Case for Feminist Revolution. New York: William Morrow and $\mathrm{Co}$.

Frank, A

1991 For a Sociology of the Body: An Analytical Review. In The Body: Social Process and Cultural Theory. Mike Featherson, Mike Hepworth, and Bryan S. Turner, eds. Pp. 36-102. London: Sage.

Georges, E.

1996 Fetal Ultrasound Imaging and the Production of Authoritative Knowledge in Greece. Medical Anthropology Quarterly 10(2):157-175.

Getz, L., and A. L. Kirkengen

2003 Uttrasound Screening in Pregnancy: Advancing Technology, Soft Markers for Fetal Chromosomal Aberrations, and Unacknowledged Ethical Dilemmas. Social Science and Medicine 56:2045-2057.

Gill, G. K.

1998 Female Feticide as a Contemporary Cultural Practice in the Punjab. Dialectical Anthropology 23(2):203-213.

Green, J. M., C. Snowdon, and H. Statham

1993 Pregnant Women's Attitudes to Abortion and Prenatal Screening. Journal of Reproductive and Infant Psychology 11:31-39.

Hanmer, Jalna

1985 Transforming Consciousness: Women and the New Reproductive Technologies. In Man-Made Women: How New Reproductive Technologies Affect Women. G. Corea, ed. Pp. 88-109. London: Hutchinson. 


\section{Happlin, Shira}

1995 Women and Obstetrics: The Loss of Childbirth to Male Physicians. Available online:

http://cyberbuzz.gatech.edu/nar/win95/shira.htm1 |february 13,2002].

Haraway, Donna

1991 Simians, Cyborgs and Women: The Reinvention of Nature. New York: Routledge.

Helitzer-Allen, D., and C. Kendall

1992 Explaining Differences between Qualitative and Quantitative Data: A Study of

Chemoprophylaxis during Pregnancy. Health Education Quarterly 19: I, 4I-5I.

Jordan, Brigitte

1993 The Achievement of Authoritative Knowledge in an American Hospital Birth. In Birth in Four Cultures: A Cross Cultural Investigation of Childbirth in Yucatan, Holland, Sweden and the United States. Brigette Jordan, ed. Pp. 151-168. Prospect Heights, IL: Waveland Press.

Kossoff, G.

1997 Contentious Issues in Safety of Diagnostic Ultrasound. Ultrasound in Obstetrics and Gynecology 10(3):151-155.

Leder, D.

1990 The Absent Body. Chicago: The University of Chicago Press.

Levi, S.

1997 The History of Ultrasound in Gynecology 1950-1980. Ultrasound in Medicine and Biology 23(4):481-552.

Liamputtong, Pranee, and Lyndsey Watson

2002 The Voices and Concerns about Prenatal Testing of Cambodian, Lao and Vietnamese Women in Australia. Midwifery 18:304-313.

Luck, C. A.

1992 Value of Routine Ultrasound Scanning at 19 Weeks: A Four-Year Study of 8,849

Deliveries. British Medical Journal 304: 1474-1478.

Lupton, Deborah

1999 Risk and the Ontology of Pregnant Embodiment. In Risk and Sociocultural Theory: New Directions and Perspectives. Deborah Lupton, ed. Pp. 59-85. Cambridge: Cambridge University Press.

Martin, Emily

1987 The Woman in the Body: A Cultural Analysis of Reproduction. Boston: Beacon Press.

Mitchell, Lisa M., and Euginia. Georges

1997 Cross-Cultural Cyborgs: Greek and Canadian Women's Discourses on Fetal U1trasound. Feminist Studies 23(2):373-401.

National Perinatal Statistics Unit

1999 Australia's Mothers and Babies 1999. Available online: http://www.npsu.unsw. eduau/Publications.htm [August 6, 200)3].

Neilson, J. P.

2000 Ultrasound for Fetal Assessment in Early Pregnancy (Cochrane Revicw). In: The Cochrane Database of Systematic Reviews, 1. Oxford: Update Software.

Novaes, H. M.

2000 Social Impacts of Technological Diffusion: Prenatal Diagnosis and Induced Abor-

tion in Brazil. Social Science and Medicine 50( I):41-51.

NVIVO Software

2002 QSR International Pty. Ltd. Melbourne, Australia.

Oakley, Ann

1984 The Trap of Medicalized Motherhood. New Society 34:639-641.

1986 The History of Ultrasonography in Obstetrics. Birth 13(1):8-13. 
Oats, J. J.

2000) Routine Antenatal Screening: A Need to Evaluate Australian Practice. Medical Journal of Australia 172(7):311-312.

O'Brien, G. D., H. P. Robinson, and P. Warren

1993 The 18 to 20 Week Obstetrical Scan. A Joint Statement from the Australasian Society for Ultrasound in Medicine, the Royal Australian College of Obstetricians and Gynaecologists and the Royal Australasian College of Radiologists. Medical Journal of Australia 158(8):567-570.

Petcheskey, Rosalind Pollack

1987 Foetal Images: The Power of Visual Culture in the Politics of Reproduction. In Reproductive Technologies: Gender, Motherhood, and Medicine. Michelle Stanworth, ed. Pp 57-80. Cambridge: Polity Press in association with B. Blackwell.

Press, Nancy, and Carol H. Browner

1997 Why Women Say Yes to Prenatal Diagnosis. Social Science and Medicine 45(7): 979-989.

RANZCOG

2001 Recommended "Best Practice" Guidelines on Antenatal Screcning for Down Syndrome and other Fetal Aneuploidy. Available online: www.ranzcog.edu.au/Open/ statements/Html/C-obs4.htm [June 17, 2002].

RANZCR

1998 Consensus Statement on the Best Use of Diagnostic Ultrasound in Obstetric Practice. Available online: www.ranzcr.cdu.au/open/pol3_1.htm [June 17, 2002].

Romero, R.

1993 Routine Obstetric Ultrasound. Ultrasound in Obstetrics and Gynaccology 3:303307.

Ruhl, Lealle

1999 Liberal Governance and Prenatal Care: Risk and Regulation in Pregnancy. Economy and Socicty 28(1):95-117.

Saari-Kcmppainen, A., O. Karjalainen, P. Ylostalo, and O. P. Heinonen

1990) Ultrasound Screening and Perinatal Mortality: Controlled Trial of Systematic One-Stage Screening in Pregnancy. The Helsinki Ultrasound Trial. Lancet 36:387-391.

Saetnan, Ann Rudinow

1996 Ultrasonic Discourse: Contested Meanings of Gender and Technology in the Norwegian Ultrasound Screening Debate. European Journal of Women's Studies 3(1): $55 \cdots 75$.

Sampson, Amanda

2000) Ultassound Snapshot: The New Schedule. Royal Australian and New Zealand College of Obstetricians and Gynaecologists 2(1):21-23.

Sheth S. S., and A. N. Malpani

1997 Inappropriate Use of New Technology: Impact on Women's Health. International Journal of Gynaccology and Obstetries 58:159-165.

Strauss, R. P

2002 Beyond Easy Answers: Prenatal Diagnosis and Counselling during Pregnancy. Cleft Palate Craniofacial Journal 39(2): 164-168.

Wade, R. V.

1999 Inages, Imagination and ldeas: $\Lambda$ Perspective on the Impact of Uitrasonography on the Practice of Obstetrics and Gynecology: Presidential Address. American Journal of Obstetrics and Gynecology 181(2):235-239.

Wajcman, Judy

1994 Delivered into Men's Hands? The Social Construction of Reproductive Technology. In Power and Decision: The Social Control of Reproduction. G. Sen and R. C. Snow, eds. Pp. 153-175. Boston: Harvard University Press. 
Weir, Lornal

1996 Recent Developments in the Government of Pregnancy. Fconomy and Society $25(3): 37.3-392$.

Willocks, J.

1996 Medical Ultrasound-A Glasgow Development which Swept the World. Avenue 19. Available online: http://www.gla.ac.uk:443/avenue/19/5medical.htm | November 5,2003 .

Woo, Joseph

2001 A Short History of the Developments of Ultrasound in Obstetrics and Gynaccology. Available online; http://www.ob-ultrasound.net/history.html |March 12, 2001 ].

Yates J. M., J. Lumley, and R. J. Bell

1995 The Prevalence and Timing of Obstetric Ultrasound in Victoria 1991-1992: A Population-Based Study. Australia New Zcaland Journal of Obstetrics and Gynaccology $35: 375-379$.

Zechmeister, I.

2001 Foetal Images: The Power of Visual Technology in Antenatal Care and the Implications for Women's Reproductive Freedom. Health Care Analysis 9(4):387-400. 